\title{
DILATED-SCALE-AWARE ATTENTION CONVNET FOR MULTI-CLASS OBJECT COUNTING
}

\author{
Wei Xu ${ }^{1 *}$, Dingkang Liang ${ }^{2 *}$, Yixiao Zheng ${ }^{1}$, Zhanyu $\mathrm{Ma}^{1 \dagger}$ \\ ${ }^{1}$ Beijing University of Posts and Telecommunications, ${ }^{2}$ Huazhong University of Science and Technology
}

\begin{abstract}
Object counting aims to estimate the number of objects in images. The leading counting approaches focus on singlecategory counting task and achieve impressive performance. Note that there are multiple categories of objects in real scenes. Multi-class object counting expands the scope of application of object counting task. The multi-target detection task can achieve multi-class object counting in some scenarios. However, it requires the dataset annotated with bounding boxes. Compared with the point annotations in mainstream object counting issues, the coordinate box-level annotations are more difficult to obtain. In this paper, we propose a simple yet efficient counting network based on point-level annotations. Specifically, we first change the traditional output channel from one to the number of categories to achieve multiclass counting. Since all categories of objects use the same feature extractor in our proposed framework, their features will interfere mutually in the shared feature space. We further design a multi-mask structure to suppress harmful interaction among objects. Extensive experiments on the challenging benchmarks illustrate that the proposed method achieves state-of-the-art counting performance.
\end{abstract}

Index Terms - Multi-Class Object Counting, PointLevel Annotation, Dilated-Scale-Aware Module, CategoryAttention Module

\section{INTRODUCTION}

Object counting is a prevalent task in the computer vision community, playing an essential role in a wide range of applications (e.g., crowd analysis, urban management, and wildlife conservation). Most counting methods count the number of a single class, such as crowd counting [1], cell counting [2], and vehicle counting [3], which can be grouped as singleclass object counting methods. Specifically, the single-class object counting methods require re-training when changing the category to be counted.

Few methods focus on counting multiple categories with a single model, and we define the problem as multi-class $o b$ ject counting. Multi-class object counting is more practical in

\footnotetext{
*Equal contribution.

† Corresponding author.
}

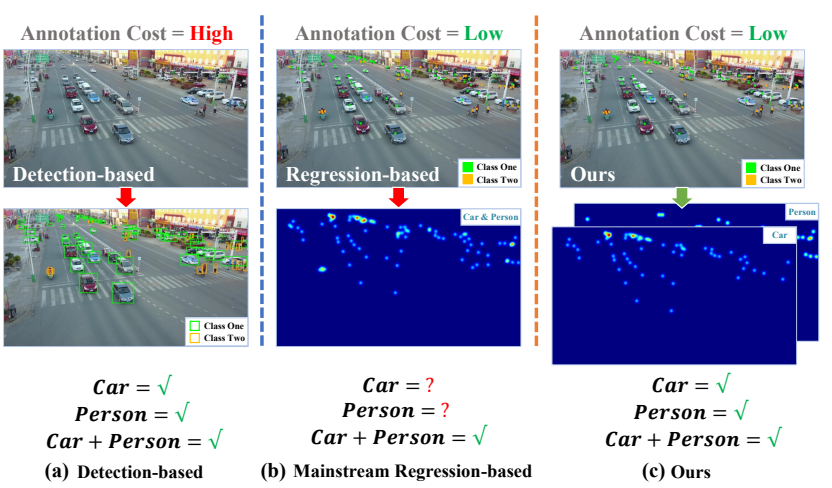

Fig. 1: Comparisons of the proposed method with regressionbased and detection-based object counting methods. (a) The detection-based methods rely on box-level annotations, but obtaining the box-level annotations in dense scenes is an expensive and labor-intensive process. (b) The mainstream regression-based methods are able to obtain the total count of all categories, but it's difficult to distinguish the number of each category. (c) The proposed method estimates density maps for each category without expensive box-level annotations.

real-world applications. For instance, it is necessary to count the appeared persons and cars in an image to assess the degree of traffic congestion during rush hour.

Detection-based methods [4, 5, 6] enable multi-class object counting tasks by counting the number of bounding boxes in each category, as shown in Fig. 1 a). However, the detection-based methods rely on bounding box annotations, which is impractical due to annotating the bounding boxes of each instance for training images can be a significant challenge in dense scenes. Thus, the mainstream counting datasets only provide point-level annotations, causing the detectors untrainable.

The regression-based methods [3, 7, 8, 9], which only rely on point-level annotations, have achieved significant successes by adopting the density maps. However, the widely used density map is obtained by overlaying a series of Gaussian Blobs. It makes the category indistinguishable so that the current regression-based counting methods hardly implement multi-class object counting, shown in Fig.[1(b).

To solve the problems mentioned above, we propose the Dilated-Scale-Aware Category-Attention ConvNet (DSACA) 
to achieve multi-class object counting, just relying on pointlevel annotations. DSACA learns multiple density maps for multi-class object counting instead of a single density map, as shown in Fig. 1 (c). Specifically, DSACA, adopting a VGG16 [10] as the backbone, consists of two crucial modules named Dilated-Scale-Aware Module (DSAM) and CategoryAttention Module (CAM), respectively. DSAM fuses the stage_3, stage_4, and stage_5 feature maps from the backbone as the input and utilizes diverse dilated rates to capture the intra-class visual responses of big objects and tiny objects simultaneously. Sharing the same backbone, the density maps will interfere with each other, which inspires us to design the CAM to provide high-quality class-isolated density maps. The main contributions of our method are summarized as follows:

1. To the best of our knowledge, this is the first attempt to implement the multi-class object counting task based on the point-level annotations in the published literature.

2. We propose a novel multi-class object counting method named DSACA consisting of DSAM and CAM. The DSAM effectively captures the multi-scale information in the intra-class scene, and the CAM shows strong adaptability to resist inter-class interference among density maps.

3. Extensive experiments demonstrate that our approach achieves state-of-the-art performance on two challenging datasets.

\section{RELATED WORKS}

The mainstream object counting methods can be categorized into detection-based methods and regression-based methods.

\subsection{Detection-based methods}

Early traditional methods capture the handcrafted features (e.g., Haar[11] and HOG[12]) to discriminate the person or non-person. These traditional methods achieve limited performance due to heavy occlusions, scale variations, and perspective distortions. Recently, the detection-based methods [4, 5, 6, 13] usually utilize deep object detectors to locate each instance since the advances of deep learning. Specifically, the pioneer RCNN [14] extracts region proposals from an image and utilizes a convolutional neural network to classify each region of interest (ROI) independently. SSD [5] is a one-stage detector, which introduces the multi-reference and multi-resolution detection fusion techniques. These deeplearning-based detectors rely on bounding-boxes annotations, but obtaining bounding-boxes annotations is an expensive and laborious process in the dense scenes.

\subsection{Regression-based methods}

Recently, various regression-based object counting methods [7, 9, 15, 16] regress a density map through different techniques such as multi-scale network [7, 17], attention mechanism [16, 18, 19], and perspective information [8, 20, 21], achieving remarkable progress. Specifically, Jiang et al. [17] introduce a novel Trellis style encoder-decoder, which effectively fuse multi-scale feature maps in the encoder. $\mathrm{Xu}$ et al. [7] propose the Learning to Scale Module (L2SM) to automatically scale different region into similar densities, reducing the pattern shift. Zhang et al. [19] propose the Attentional Neural Field (ANF), which combines the conditional random fields with non-local attention mechanisms to capture multiscale features and long-range dependencies. Yang et al. [8] propose a reverse perspective network, which is designed to diminish the scale variations in an unsupervised way. Liu et al. [21] utilize a auxiliary branch to integrate perspective maps into the density maps.

Regression-based methods usually adopt MSE loss as their loss function. However, only use the MSE loss function will cause some problems, such as blur effect, neglecting the local consistency, and losing position information. Therefore, designing an appropriate loss function is also an essential researching direction in the training stage, which can promote the object counting ability. BL [15] regards the density map as a probability map, computing the probability of each pixel. SPANet [22] proposes Maximum Excess over Pixels (MEP) loss by finding the pixel-level subregion with the highest discrepancy with ground truth.

In general, these regression-based methods only focus on single class object counting (e.g., crowd counting), which limits the applications in the real-world.

\section{PROPOSED METHOD}

The overview pipeline of our method is shown in Fig. 2. The proposed method mainly contains two crucial components: the Dilated-Scale-Aware Module (DSAM) and CategoryAttention Module (CAM). We utilize the pre-trained VGG16 as the backbone and discard the pooling layer between the stage_4 and stage_5 to maintain the spatial size, which follows previous works [7, 8, 18]. The backend utilizes $3 \times 3$ convolutional layers and a $1 \times 1$ layer to generate the density maps. The DSAM is designed to capture the visual context cues by extracting features at different scales. The CAM focus on the inter-class feature decorrelation, which can effectively reduce the bad interference among density maps belonging to different categories.

\subsection{Multi-Class object counting problem definition}

The current single-class object counting methods apply the Gaussian kernels on point annotations to generate density maps as ground truth and make predictions about the number of a single class. To realize the multi-class object counting, 


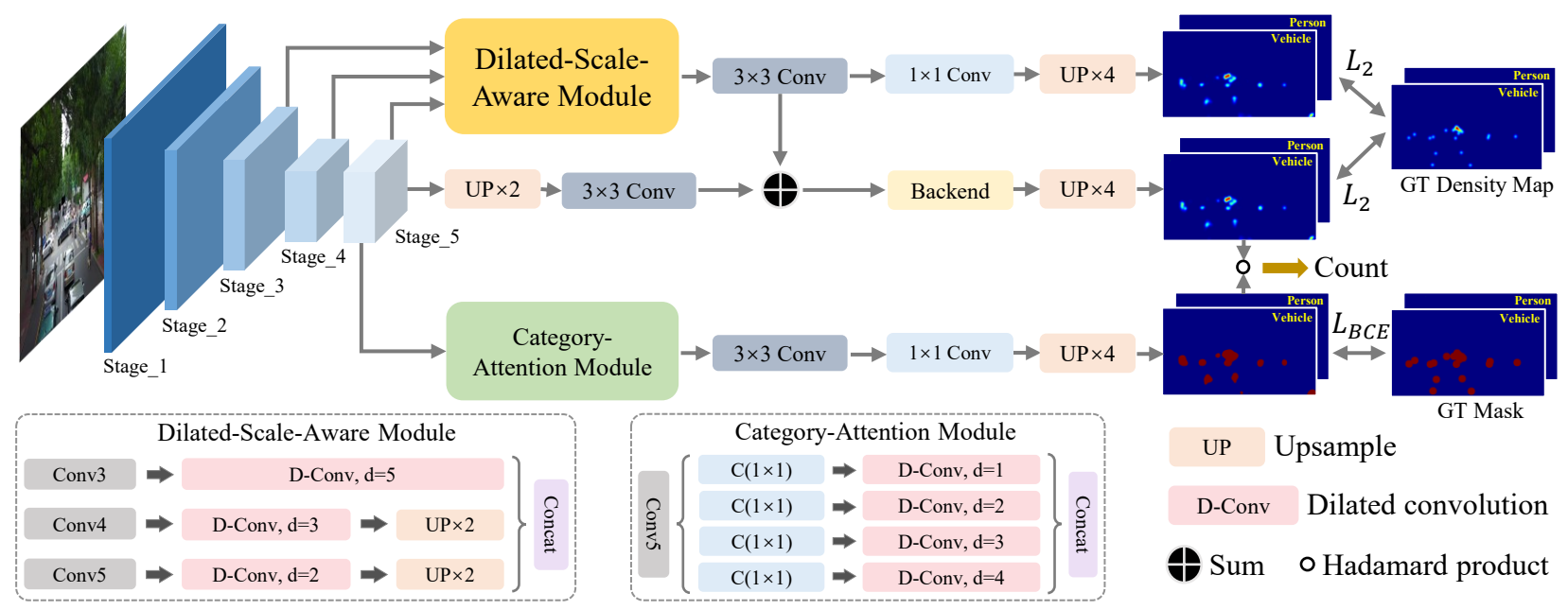

Fig. 2: The pipeline of our method. The proposed approach fuse multi-scale features via Dilated-Scale-Aware Module and suppress harmful inter-class responses via Category-Attention Module.

we generate individual density maps for each category in an image. To be specific, given an image containing $N$ classes of objects, the ground-truth density map $D$ can be illustrated as:

$$
D=\left\{\begin{array}{c}
\sum_{i=1}^{C_{1}} \delta\left(P^{1}-P_{i}^{1}\right) * G_{\sigma^{1}}\left(P^{1}\right) \\
\vdots \\
\sum_{i=1}^{C_{k}} \delta\left(P^{k}-P_{i}^{k}\right) * G_{\sigma^{k}}\left(P^{k}\right), \\
\vdots \\
\sum_{i=1}^{C_{n}} \delta\left(P^{n}-P_{i}^{n}\right) * G_{\sigma^{n}}\left(P^{n}\right)
\end{array}\right.
$$

where $P_{i}^{k}$ represents the point annotation of the $i$-th instance belonging to the $k$-th class. There are $C_{k}$ instances in the $k$ th class. $\sigma^{k}$ is the kernel size for the $k$-th class in Gaussian function $G$, and the value of $\sigma^{k}$ depends on the relative sizes of the $k$-th class.

\subsection{Dilated-Scale-Aware Module}

Due to the perspective effect, the scales of objects change a lot in a scene. It forms a challenge for feature extraction. To overcome this issue, we propose the Dilated-ScaleAware Module (DSAM) that captures multi-scale information by fusing features from different layers of the backbone.

In the VGG16 with max-pooling layers, the receptive field of convolution layers become progressively larger as the depth increases. The intermediate layers of a CNN hierarchically learn visual patterns from edges and corners to parts and objects. As shown in Fig. 2, the proposed DSAM applies different dilated convolutions on stage_3, stage_4 and stage_5. The expansion ratios of dilated convolutional filters are set as 2,3 , and 5, respectively. After the upsample and concatenation operation, a $3 \times 3$ convolutional layer is added to fuse these multi-scale features. By doing so, the DSAM effectively combines hierarchical features from different layers, which indeed improves the object counting performance.

\subsection{Category-Attention Module}

Although the DSAM effectively mitigates the effect of intraclass scale variation, mutual interference between classes exits on the density maps. This inspires us to design the Category-Attention Module (CAM) to generate the discriminative density maps, as shown in Fig. 2 .

The CAM is designed to generate spatial attentions for each class. We generate the pseudo ground-truth mask maps for each instance from point annotations to supervise the learning of spatial attentions. Specifically, we first utilize the distance transformation [25] and transform the point annotation map to a distance map $D$, which is described as:

$$
P_{(x, y)}=\min _{\left(x_{i}, y_{i}\right) \in A} \sqrt{\left(x-x_{i}\right)^{2}+\left(y-y_{i}\right)^{2}}
$$

where $P_{(x, y)}$ represents the pixel value located at $(x, y)$ in the distance map $D$. $A$ is the set of annotated coordinates. The pseudo-labels for spatial attention generation can be obtained as a $0-1$ mask by using a distance threshold $J$ to divide the distance map into the foreground and background.

As shown in Fig. 2, the CAM adopts the feature maps of stage 5 as input since the high-level layer learns more semantic information. Similar to the DSAM, the CAM enhances the multi-scale information via four dilated convolutional layers with 1, 2, 3, 4 dilation rates, respectively. The multi-scale feature maps are combined via channel-wise concatenation operation and several convolution layers. The number of the spatial attentions is equal to the number of categories, and the estimated spatial attentions multiplies the estimated density maps to obtain the final density maps, which effectively reduces the inter-class interference. 

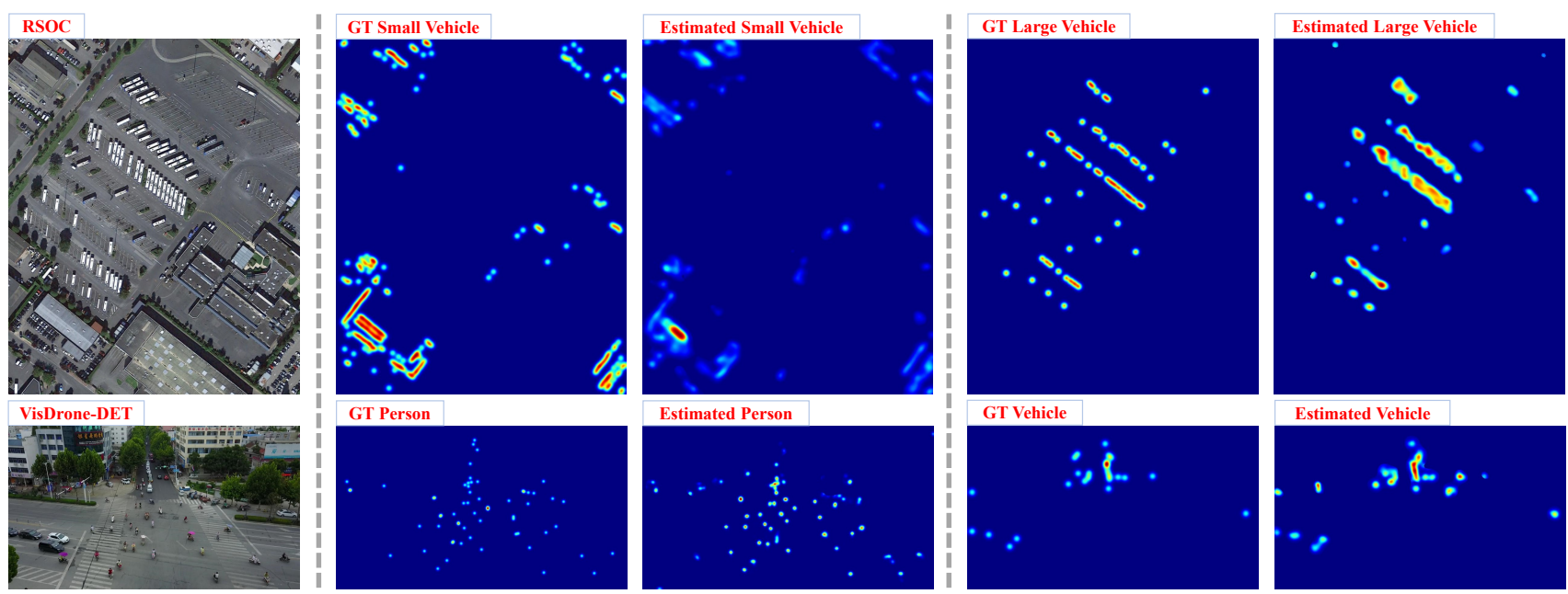

Fig. 3: Visualization results on the RSOC dataset [23] and the VisDrone-DET dataset [24]. The given images of the two datasets contain huge differences in viewpoint, scene, target size, and image scale, but our method shows strong robustness as well as impressive multi-class object counting performance.

\subsection{Loss function}

We utilize the $L_{2}$ loss to measure the difference between the ground truth density maps and estimated density maps:

$$
L_{2}=\sum_{n=1}^{N} \sum_{x=1}^{W} \sum_{y=1}^{H}\left|P_{(n, x, y)}^{\prime}-P_{(n, x, y)}\right|^{2},
$$

where $N$ is the total number of the categories. $W$ and $H$ represent the width and height of the training image. $P_{(n, x, y)}^{\prime}$ and $P_{(n, x, y)}$ represent the pixel values located at $(x, y)$ in the estimated density map and the ground-truth density map of the $c$-th class respectively.

We train the CAM based on the Binary Cross Entropy loss, as defined by:

$$
\begin{aligned}
L_{B C E}= & -\frac{1}{W \times H} \sum_{x=1}^{W} \sum_{y=1}^{H}\left(\left(T_{(x, y)} \times \log R_{(x, y)}\right)\right. \\
& \left.+\left(1-T_{(x, y)}\right) \times \log \left(1-R_{(x, y)}\right)\right),
\end{aligned}
$$

where $T_{(x, y)} \in\{0,1\}$ denotes the pseudo-label for spatial attention located at $(x, y) . R_{(x, y)} \in[0,1]$ represents the predicted spatial attention. The final training objective function $L$ is defined as below:

$$
L=\sum_{i=1}^{2} L_{2}^{i}+\sum_{n=1}^{N} L_{B C E}^{n}
$$

\section{EXPERIMENTS}

\subsection{Implementation details}

The proposed network is trained in an end-to-end fashion. For the first 13 convolutional layers, we initialize them with a pretrained VGG-16 [10]. The other layers are initialized by a

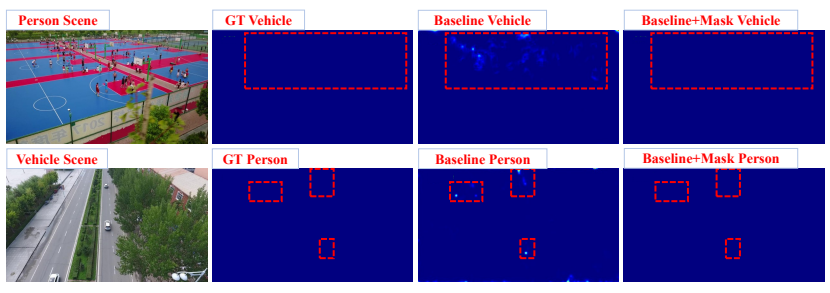

Fig. 4: In the person-vehicle counting task, both estimated density maps are interfered by each other, as shown in the red dashed boxes. The spatial attention masks suppressed these harmful responses effectively.

Gaussian initialization with 0.01 standard deviation. The distance threshold $J$ is set as 20 . We flip each image and crop the given images randomly as the data augmentation processes. The parameters are updated by an adaptive moment estimation optimizer.

\subsection{Datasets}

Since there are no publicly available multi-class object counting datasets, we utilize the single-class object counting dataset RSOC [23] including multiple subsets and the object detection dataset VisDrone-DET [24] for evaluation. Note that object detection datasets provide box-level annotations instead of point-level annotations. We obtain the point-level annotations by computing the center of bounding boxes. All the experiments are conducted on the point-level annotations.

VisDrone-DET Dataset [24]. This dataset contains 10,209 images captured by cameras equipped on drones. There are ten categories annotated, including pedestrian, person, car, van, bus, truck, motor, bicycle, awning-tricycle, and tricycle. To increase the density of the counting instances and ensure the challenge for counting task, we merge the pedes- 
Table 1: Performance comparisons on the VisDrone-DET dataset [24]. The first and second places are marked in red and blue respectively.

\begin{tabular}{l|c|c|c|c|c|c|c}
\hline \multirow{2}{*}{ Method } & \multirow{2}{*}{ Backbone } & \multicolumn{2}{|c|}{ Mean } & \multicolumn{2}{c|}{ Vehicle } & \multicolumn{2}{c}{ Person } \\
\cline { 3 - 8 } & & MAE & MSE & MAE & MSE & MAE & MSE \\
\hline MCNN [9] & - & 9.664 & 14.123 & 8.808 & 11.885 & 10.520 & 16.361 \\
SANet [27] & - & 16.807 & 24.091 & 17.992 & 23.234 & 15.623 & 24.947 \\
CSRNet [28] & VGG16 & 6.778 & 9.429 & 6.022 & 8.091 & 7.535 & 10.767 \\
BL [15] & VGG19 & 7.503 & 10.504 & 6.754 & 9.021 & 8.252 & 11.988 \\
CAN [21] & VGG16 & 6.224 & 8.831 & 5.662 & 7.667 & 6.786 & 9.994 \\
\hline Ours & VGG16 & 4.030 & 6.285 & 3.437 & 5.468 & 4.624 & 7.102 \\
\hline
\end{tabular}

Table 2: Performance comparisons on the RSOC dataset [23]. The first and second places are marked in red and blue respectively.

\begin{tabular}{l|c|c|c|c|c|c|c}
\hline \multirow{2}{*}{ Method } & \multirow{2}{*}{ Backbone } & \multicolumn{2}{|c|}{ Mean } & \multicolumn{2}{c|}{ Small-Vehicle } & \multicolumn{2}{c}{ Large-Vehicle } \\
\cline { 3 - 8 } & & MAE & MSE & MAE & MSE & MAE & MSE \\
\hline MCNN [9] & - & 105.600 & 425.029 & 186.913 & 814.102 & 24.288 & 35.957 \\
SANet [27] & - & 143.670 & 500.228 & 246.830 & 939.159 & 40.509 & 61.298 \\
CSRNet [28] & VGG16 & 87.537 & 361.727 & 154.898 & 697.101 & 20.177 & 26.353 \\
BL [15] & VGG19 & 107.212 & 297.607 & 172.883 & 534.460 & 41.541 & 60.754 \\
CAN [21] & VGG16 & 58.987 & 137.238 & 94.772 & 235.974 & 23.201 & 38.501 \\
\hline Ours & VGG16 & 42.432 & 127.441 & 65.398 & 223.578 & 19.467 & 31.305 \\
\hline
\end{tabular}

trian category into the person category and consider car, van, bus, truck as the same category named vehicle. The extremely small size of objects is the major challenge of this dataset.

RSOC Dataset [23]. This dataset contains 3, 057 images collected from Google Earth and DOTA dataset [26]. It is the largest object counting dataset collected in a remote sense. And the instances included are in high density. There are four categories of images, including buildings, small vehicles, large vehicles, and ships. Specifically, the small vehicles and the large vehicles always appear in the same image, which meets the labeling requirements for multi-class object counting tasks. In our experiments, we choose the small vehicle and large vehicle subsets into a multi-class object counting dataset. The most challenging issue of the RSOC dataset is the severe background noise.

\subsection{Evaluation metrics}

The Mean Absolute Error (MAE) and the Mean Squared Error (MSE) are the primary metrics in the object counting task. In the multi-class counting task, we adopt MAE and MSE to evaluate counting performance for each class, then average the results of all the categories to get $M A E_{\text {Mean }}$ and $M S E_{\text {Mean }}$, which denote the multi-class counting capability:

$$
\begin{aligned}
& M A E=\frac{1}{M} \sum_{m=1}^{M}\left|Q_{m}^{\prime}-Q_{m}\right|, M S E=\sqrt{\frac{1}{M} \sum_{m=1}^{M}\left(Q_{m}^{\prime}-Q_{m}\right)^{2}} \\
& M A E_{\text {Mean }}=\frac{1}{N} \sum_{n=1}^{N} M A E_{n}, M S E_{\text {Mean }}=\frac{1}{N} \sum_{n=1}^{N} M S E_{n}
\end{aligned}
$$

where $M$ denotes the number of images in the testing set, $N$ is the number of the categories. $Q_{m}^{\prime}$ and $Q_{m}$ are the estimated and labeling count number of the $m$-th image.
Table 3: Ablation studies on the VisDrone-DET dataset [24]. The first and second places are marked in red and blue respectively.

\begin{tabular}{l|c|c|c|c|c|c}
\hline \multirow{2}{*}{ Method } & \multicolumn{2}{|c|}{ Mean } & \multicolumn{2}{c|}{ Vehicle } & \multicolumn{2}{c}{ Person } \\
\cline { 2 - 7 } & MAE & MSE & MAE & MSE & MAE & MSE \\
\hline baseline & 6.823 & 9.549 & 6.242 & 8.363 & 7.405 & 10.736 \\
baseline+DSAM & 5.066 & 7.439 & 4.233 & 6.049 & 5.900 & 8.828 \\
baseline+CAM & 4.202 & 6.649 & 3.556 & 5.455 & 4.849 & 7.844 \\
baseline+DSAM+CAM & 4.030 & 6.285 & 3.437 & 5.468 & 4.624 & 7.102 \\
\hline
\end{tabular}

\subsection{Comparisons with the State-of-the-art}

The traditional object counting methods are designed for single-class object counting task. While for multi-class object counting task, the previous point-level annotations based object counting methods should train separate models for each category. The experiments on these two datasets are twocategory object counting. For fair comparisons, we change the output channel of other methods to 2 to implement the multi-class object counting task. Tab. 1 and Tab. 2 list the quantitative results of VisDrone-DET [24] and RSOC [23] respectively, and we also tell the quality of estimated density maps in Fig. 3

VisDrone-DET Dataset. We first compare our method with various classic object counting methods on the VisDrone-DET dataset. The proposed method achieves the best performance on all metrics. Specially, our method improves $35.3 \%$ in $M A E_{\text {Mean }}, 28.8 \%$ in $M S E_{\text {Mean }}, 39.3 \%$ in $M A E_{\text {Vehicle }}, 28.7 \%$ in $M S E_{\text {Vehicle }}, 31.9 \%$ in $M A E_{\text {Person }}$ and $28.9 \%$ in $M S E_{\text {Person }}$ compared with CAN.

RSOC Dataset. We also compare our method with other related works on the RSOC dataset. As we can see, the proposed method achieves 42.432 in $M A E_{\text {Mean }}$ and 127.441 in $M S E_{\text {Mean }}$, which is currently the best multi-class object counting performance. Counting the Small-Vehicle, our approach improves $31.0 \%$ in $M A E_{\text {Small-Vehicle }}$ and achieves 12.396 MS $E_{\text {Small-Vehicle }}$ dropped compared with CAN, respectively.

In summary, the proposed method shows outstanding performance on these two datasets.

\subsection{Ablation Studies}

In this section, we conduct the ablation studies to verify the effectiveness of DSAM and CAM on the VisDrone-DET dataset [24].

Effectiveness of DSAM. We first study the impact of DSAM, which is designed to capture the intra-class multiscale information. The results are listed in Tab. 3. We find that the addition of DSAM causes $1.757 M A E_{\text {Mean }}$ and 2.11 $M S E_{\text {Mean }}$ dropped compared with the baseline.

Effectiveness of CAM. We further validate the effect of the proposed CAM. The results are listed in Tab. 3. CAM achieves $38.4 \% M A E_{\text {Mean }}$ and $30.4 \%$ MS $E_{\text {Mean }}$ improvement, which indicates that CAM effectively reduces the interclass interference on density maps. By observing Fig. 4, we 
can see that the predicted results with CAM exhibit better clarity and better accuracy compared with baseline in both crowd scenes and vehicle scenes.

\section{CONCLUSION}

In this paper, we propose a novel Dilated-Scale-Aware Category-Attention ConvNet (DSACA), which achieves multi-class object counting simultaneously only based on point-level annotations. To overcome the inter-class interference that appears among the density maps and mitigate the effects of intra-class scale variations, we designed DilatedScale-Aware Module (DSAM) and Category-Attention Module (CAM). Extensive experiments on two challenging benchmarks show the validity of our approach. In the future work, we will explore the correlation of multi-class object counting and single-class object counting systematically.

\section{References}

[1] Q. Wang, J. Gao, W. Lin, and X. Li, "Nwpu-crowd: A large-scale benchmark for crowd counting and localization," IEEE Transactions on Pattern Analysis and Machine Intelligence, 2020.

[2] T. Falk, D. Mai, R. Bensch, Ö. Çiçek, A. Abdulkadir, Y. Marrakchi, A. Böhm, J. Deubner, Z. Jäckel, K. Seiwald, et al., "U-net: deep learning for cell counting, detection, and morphometry," Nature methods, 2019.

[3] R. Guerrero-Gómez-Olmedo, B. Torre-Jiménez, R. López-Sastre, S. Maldonado-Bascón, and D. OnoroRubio, "Extremely overlapping vehicle counting," in IbPRIA, 2015.

[4] S. Ren, K. He, R. Girshick, and J. Sun, "Faster r-cnn: Towards real-time object detection with region proposal networks," IEEE transactions on pattern analysis and machine intelligence, 2016.

[5] W. Liu, D. Anguelov, D. Erhan, C. Szegedy, S. Reed, C.-Y. Fu, and A. C. Berg, "Ssd: Single shot multibox detector," in $E C C V, 2016$.

[6] J. Redmon, S. Divvala, R. Girshick, and A. Farhadi, "You only look once: Unified, real-time object detection," in CVPR, 2016.

[7] C. Xu, K. Qiu, J. Fu, S. Bai, Y. Xu, and X. Bai, "Learn to scale: Generating multipolar normalized density map for crowd counting," ICCV, 2019.

[8] Y. Yang, G. Li, Z. Wu, L. Su, Q. Huang, and N. Sebe, "Reverse perspective network for perspective-aware object counting," in CVPR, 2020.

[9] Y. Zhang, D. Zhou, S. Chen, S. Gao, and Y. Ma, "Singleimage crowd counting via multi-column convolutional neural network," in CVPR, 2016.

[10] K. Simonyan and A. Zisserman, "Very deep convolutional networks for large-scale image recognition," international conference on learning representations, 2015.

[11] C. P. Papageorgiou, M. Oren, and T. Poggio, "A general framework for object detection," in ICCV, 1998.

[12] N. Dalal and B. Triggs, "Histograms of oriented gradients for human detection," in CVPR, 2005.

[13] X. Zhou, D. Wang, and P. Krähenbühl, "Objects as points," arXiv preprint arXiv:1904.07850, 2019.

[14] R. Girshick, J. Donahue, T. Darrell, and J. Malik, "Rich feature hierarchies for accurate object detection and semantic segmentation," in CVPR, 2014.

[15] Z. Ma, X. Wei, X. Hong, and Y. Gong, "Bayesian loss for crowd count estimation with point supervision," in ICCV, 2019.

[16] N. Liu, Y. Long, C. Zou, Q. Niu, L. Pan, and H. Wu, "Adcrowdnet: An attention-injective deformable convolutional network for crowd understanding," in CVPR, 2019.

[17] X. Jiang, Z. Xiao, B. Zhang, X. Zhen, X. Cao, D. Doermann, and L. Shao, "Crowd counting and density estimation by trellis encoder-decoder networks," in CVPR, 2019.

[18] S. Bai, Z. He, Y. Qiao, H. Hu, W. Wu, and J. Yan, “Adaptive dilated network with self-correction supervision for counting," in CVPR, 2020.

[19] A. Zhang, L. Yue, J. Shen, F. Zhu, X. Zhen, X. Cao, and L. Shao, "Attentional neural fields for crowd counting," in ICCV, 2019.

[20] Z. Yan, Y. Yuan, W. Zuo, X. Tan, Y. Wang, S. Wen, and E. Ding, "Perspective-guided convolution networks for crowd counting," in ICCV, 2019.

[21] W. Liu, M. Salzmann, and P. Fua, "Context-aware crowd counting," in CVPR, 2019.

[22] Z.-Q. Cheng, J.-X. Li, Q. Dai, X. Wu, and A. G. Hauptmann, "Learning spatial awareness to improve crowd counting," in ICCV, 2019.

[23] G. Gao, Q. Liu, and Y. Wang, "Counting from sky: A large-scale data set for remote sensing object counting and a benchmark method," IEEE Transactions on Geoscience and Remote Sensing, 2020.

[24] P. Zhu, L. Wen, D. Du, X. Bian, H. Ling, Q. Hu, H. Wu, Q. Nie, H. Cheng, C. Liu, et al., "Visdrone-vdt2018: The vision meets drone video detection and tracking challenge results," in ECCV, 2018.

[25] A. Rosenfeld and J. L. Pfaltz, "Distance functions on digital pictures," Pattern recognition, 1968.

[26] G.-S. Xia, X. Bai, J. Ding, Z. Zhu, S. Belongie, J. Luo, M. Datcu, M. Pelillo, and L. Zhang, "Dota: A largescale dataset for object detection in aerial images," in CVPR, 2018.

[27] X. Cao, Z. Wang, Y. Zhao, and F. Su, "Scale aggregation network for accurate and efficient crowd counting," in ECCV, 2018. 
[28] Y. Li, X. Zhang, and D. Chen, "Csrnet: Dilated convolutional neural networks for understanding the highly congested scenes," in CVPR, 2018. 